\title{
Study on Frequency Stability of Magnetic Resonance Contactless Power Transmission System Based On PSSS Topology
}

\author{
Xin Zhao ${ }^{1,}$, Hai Fu Ye , b $^{1}$ \\ ${ }^{1}$ Institute of Electronic Engineering, China Academy of Engineering Physics, Mianyang 621900, \\ China \\ azhaoxin881028@163.com, byehaifu@126.com
}

\begin{abstract}
Keywords: Wireless Power Transfer, Magnetic Resonance, Dynamic Resonance, Constant Frequency Control
\end{abstract}

Abstract. This paper, aimed at the magnetic resonance contactless power transmission system based on PSSS topology, analyzes the stability of the system resonant frequency when the load and the mutual inductance are changing and proposes the method of constant frequency control based on the technology of dynamic resonance and soft switching. The paper explains the operating principles of the proposal, builds the circuit model by using Simulink and conducts a simulation study.

\section{Introduction}

Compared to traditional inductive contactless power transmission technology, magnetic resonance contactless power transmission technology can generate a higher magnetic field so that the power can be transferred more efficiently at a farther distance. Magnetic resonance contactless power transmission technology is a kind of technology which is non-directional and can transfer several kilowatts of power efficiently at medium distance (The medium distance is more than a few times the diameter of the resonance coils) through non-metallic materials, having a relatively small effect on the human body and the environment [1]- [3].

Fig. 1 shows the schematic diagram of resonance contactless power transmission system based on PSSS topology. The coupling coils of the system consists of a transmitting coil, resonance coil 1, resonance coil 2 and a pick-up coil. $C_{p} 、 C_{l} 、 C_{2}$ and $C_{s}$ are compensation capacitors which make the system work in resonant state by compensating the reactive power generated by the coupling coils. Therefore, to maximize the power transmission efficiency of the whole system, the inherent resonance frequency of each coupling coil must be consistent. However, the system load or coil mutual inductance can be affected by some factors in practical application, which makes the reflection impedance of the primary side change. In this way, the coupling coils are dissonant due to the inherent resonant frequency and the transmission power of the system is decreased[4,5].

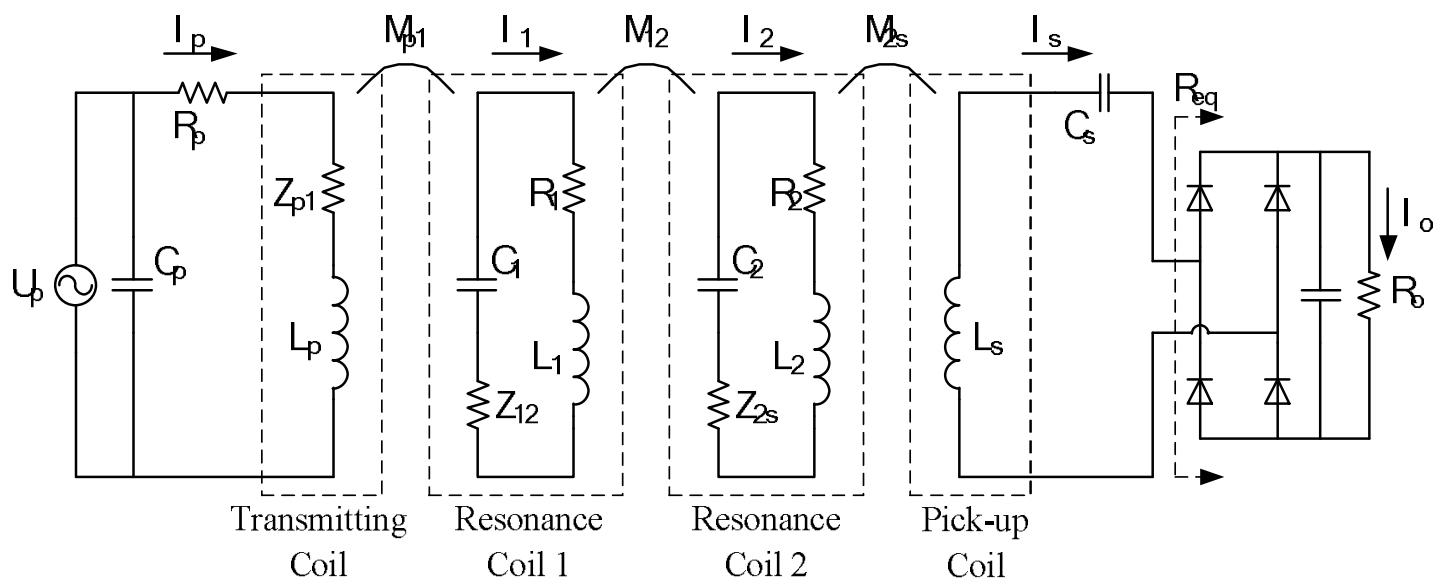

Fig. 1 The schematic diagram of resonance contactless power transmission system based on PSSS topology

In the case of the dissonance of the transmitting coil, the soft switching technology is usually adopted. But the characteristic of soft switch floating-frequency control can lead to the resonance coils 
and the pick-up coil dissonance. Therefore, in the case of the dynamic change of the magnetic resonance system load or coil mutual inductance, the frequency of the system should be kept stable[6,7]. At present, the mainstream frequency stabilization control strategy is based on dynamic compensation, including dynamic capacitor compensation and phase control inductance. Dynamic capacitor compensation is limited by the dispersion of capacitive value, so the adjusting range is limited and the control is not easy to achieve; And a single control strategy based on phase control inductance is focused on tuning the inherent resonant frequency of resonant network, ignoring the power loss under the high frequency switching condition.

This paper, aimed at the magnetic resonance contactless power transmission system based on PSSS topology, analyzes the influence of system load and the coil mutual inductance on the inherent resonance frequency of system primary side, proposes a method of constant frequency control based on the technology of dynamic resonance and soft switching which achieves the stability of the inherent resonant frequency of coupling coils, and then builds the circuit model by using Simulink and conducts a simulation study.

\section{The analysis of the resonance frequency of primary side}

As shown in Fig. $1, U_{p}$ is the effective value of AC output voltage of high frequency inverter circuit, $Z_{p 1}$ is the equivalent reflection impedance from resonance coil $L_{1}$ to transmitting coil $L_{p}, Z_{12}$ is the equivalent reflection impedance from resonance coil $L_{2}$ to resonance coil $L_{1}, Z_{2 s}$ is the equivalent reflection impedance from pick-up coil $L_{s}$ to resonance coil $L_{2}$. And $M_{p 1}$ represents the mutual inductance between transmitting coil $L_{p}$ and resonance coil $L_{1}, M_{12}$ represents the mutual inductance between resonance coil $L_{1}$ and resonance coil $L_{2}, M_{2 s}$ represents the mutual inductance between resonance coil $L_{2}$ and pick-up coil $L_{s}$. In order to simplify the analysis, the radiation loss and the ohmic loss of the coils are ignored. According to the theory of mutual inductance, when all the coupling coils work in resonance state, equations can be obtained as follows:

$$
\left\{\begin{array}{l}
Z_{p 1}=\frac{\omega_{o}^{2} M_{p 1}^{2}}{Z_{12}} \\
Z_{12}=\frac{\omega_{o}^{2} M_{12}^{2}}{Z_{2 s}} \\
Z_{2 s}=\frac{\omega_{o}^{2} M_{2 s}^{2}}{R_{e q}} \\
R_{e q}=\frac{8}{\pi^{2}} R_{o} \\
C_{p}=\frac{L_{p}}{\omega_{o}^{2} L_{p}^{2}+Z_{p 1}^{2}} .
\end{array}\right.
$$

Then the following equation can be derived:

$$
\frac{\pi^{4} \omega_{p}^{4} M_{p 1}^{4} M_{2 s}^{4}}{64 M_{12}^{4} R_{o}^{2}} \omega_{p}^{4}+L_{p}^{2} \omega_{p}^{2}-\frac{L_{p}}{C_{p}}=0 .
$$

Substituting the result of Eq. 2 to $\omega=2 \pi \mathrm{f}$ can give

$$
f_{p}=\sqrt{\frac{8 M_{12}^{4} R_{o}^{2}\left(\sqrt{L_{p}^{4}+\frac{\pi^{4} L_{p} M_{p 1}^{4} M_{2 s}^{4}}{16 C_{p} M_{12}^{4} R_{o}^{2}}}-L_{p}^{2}\right)}{\pi^{6} M_{p 1}^{4} M_{2 s}^{4}} .}
$$

And $\mathrm{f}_{\mathrm{p}}$ is the inherent resonant frequency of transmitting coil resonant network. 
In practical application, the relative position of transmitting coil $L_{p}$ and resonance coil $L_{l}$ is fixed, so the mutual inductance $M_{p 1}$ can be regarded as a constant. Also, the mutual inductance $M_{2 s}$ can be regarded as a constant. Therefore, the paper will focus on the influence of changing mutual inductance $M_{12}$ and system load $R_{o}$ on $f_{p}$. As Eq. 3 shows, $f_{p}$ will obviously change with the change of $R_{o}$ and $M_{12}$. And they have a nonlinear relationship. Table 1 lists a set of parameters, where the change range of $R_{o}$ is from $1.5 \Omega$ to $10 \Omega$ and the change range of $M_{12}$ is from $0.05 \mu \mathrm{H}$ to $0.5 \mu \mathrm{H}$.

Table 1 System Parameters

\begin{tabular}{c|c}
\hline \hline Parameters & Value \\
\hline$M_{p 1}$ & $0.61[\mu \mathrm{H}]$ \\
\hline$M_{2 s}$ & $0.47[\mu \mathrm{H}]$ \\
\hline$L_{p}$ & $10[\mu \mathrm{H}]$ \\
\hline$L_{s}$ & $10[\mu \mathrm{H}]$ \\
\hline$f$ & $1[\mathrm{MHz}]$ \\
\hline$R_{o}$ & $2[\Omega]$ \\
\hline$M_{12}$ & $0.2[\mu \mathrm{H}]$ \\
\hline
\end{tabular}

Substituting the parameters in Table 1 to Eq. 3, the relationship between $f_{p}$ and $M_{12}$ and $R_{o}$ can be obtained, as shown in Fig. 2.

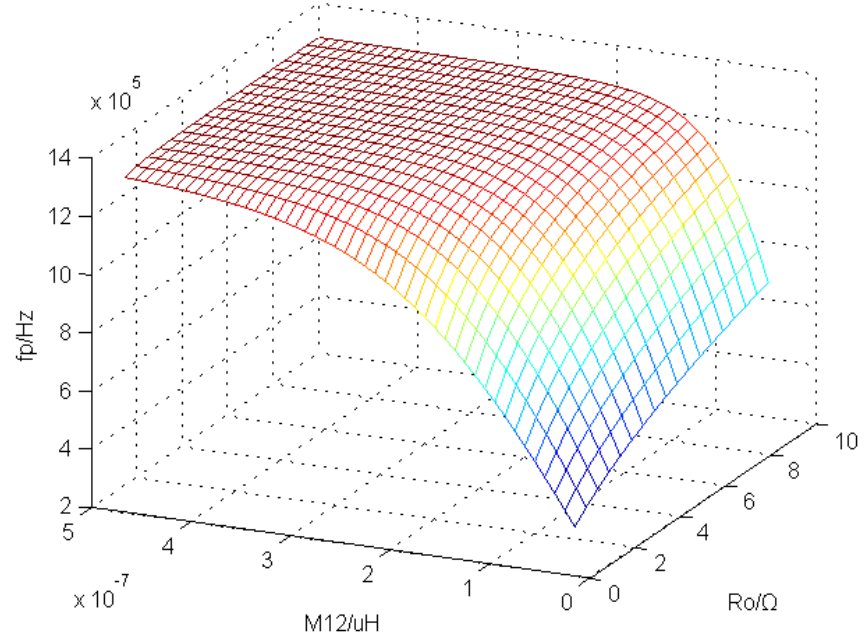

Fig. 2 The relation curve of $f_{p}$ with $M_{12}$ and $R_{o}$

Fig. 2 shows that $f_{p}$ will change dramatically with the change of $M_{12}$ and $R_{o}$ in a certain range. Since the frequency of the magnetic resonance contactless power transmission system is usually a few $\mathrm{MHz}$ or even higher, even a small frequency drift can greatly attenuate the transmission power of the system. Appropriate control is needed to ensure the stability of the system's inherent resonant frequency.

\section{Constant frequency control based on the technology of dynamic resonance and soft switching}

The principle of dynamic resonance based on phase control inductance. The schematic diagram of the phase control inductance branch circuit is shown in Fig. 3, which consists of a fixed inductive Lt and two opposite switches $S_{t 1}$ and $S_{t 2}$, and form the resonant compensation circuit of the primary side together with $C_{p}$. The circuit can change the conduction angle of $\mathrm{Lt}$ in the positive or negative half period by controlling $S_{t 1}$ and $S_{t 2}$ respectively to change the equivalent impedance of the resonant compensation circuit. Thus, the inherent resonance frequency of system primary side can be changed. 


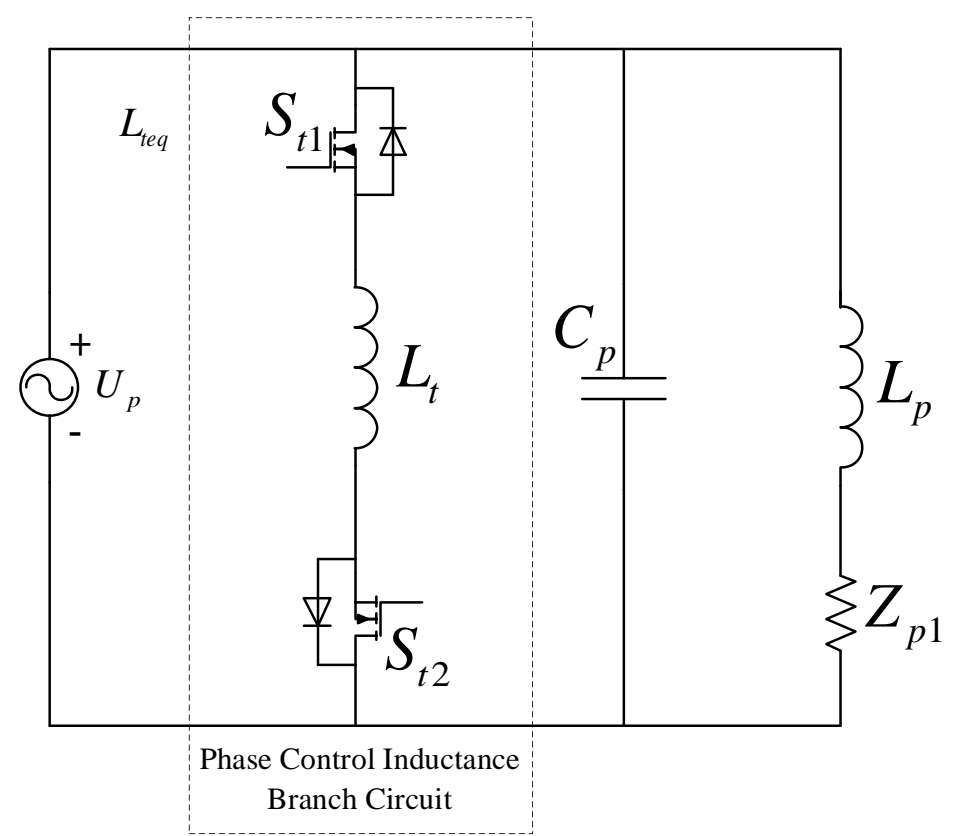

Fig. 3 The schematic diagram of phase control inductance branch circuit As shown in Fig. 3, $u_{p}$ is the input voltage of the circuit which is sinusoidal and defined as $u_{p}=\sqrt{2} U_{p} \sin (\omega t)$,

where $U_{p}$ is the effective value of the input voltage. Fig. 4 shows voltage and current waveform of $L_{t}$ when internal resistance of $L_{t}$ is ignored and $\alpha>\pi / 2$ where $\alpha$ represents the trigger angle of switches. Since the energy stored by the inductor is equal to the energy released by it, the conduction angle of switches is $2 \pi-2 \alpha$.

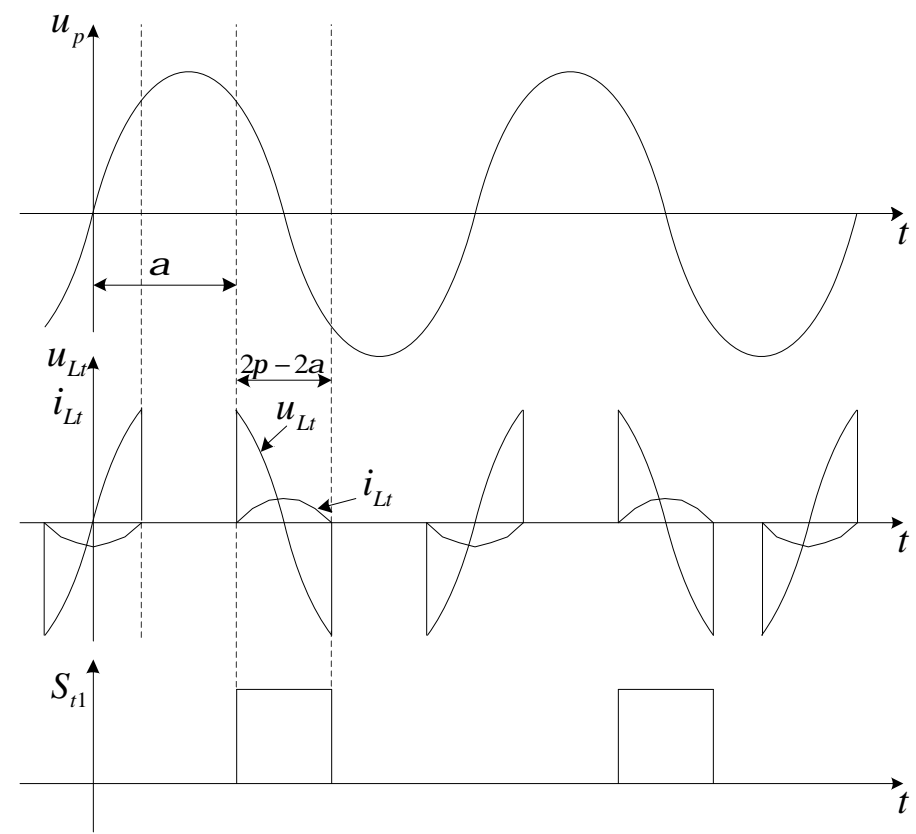

Fig. 4 The voltage and current waveform of $L_{t}$

By expanding the voltage of $L_{t}$ with the form of Fourier series, $U_{L t}$, which represents the fundamental component of the voltage of $L_{t}$, can be derived:

$$
U_{L t}=\frac{\sqrt{2} U_{p}}{\pi}(2 \pi-2 \alpha+\sin 2 \alpha) \cos \omega t .
$$

And the effective values of $U_{L t}$ and the fundamental component of the current of $L_{t}$, represented by $U_{L t R M S}$ and $I_{L t R M S}$ respectively, are 


$$
\left\{\begin{array}{l}
U_{L t R M S}=\frac{U_{p}}{\pi}(2 \pi-2 \alpha+\sin 2 \alpha) \\
I_{L t R M S}=\frac{U_{L t R M S}}{\omega L_{t}}
\end{array} .\right.
$$

$L_{t e q}$ represents the equivalent inductance of the inductance phase controlling branch circuit. When the trigger angle is $\alpha, L_{\text {teq }}$ is

$$
L_{\text {teq }}=\frac{U_{p}}{\omega I_{L t R M S}}=\frac{\pi L_{t}}{2 \pi-2 \alpha+\sin 2 \alpha} .
$$

According to Eq. 7, it can be obtained that, when the change range of $\alpha$ is from $\pi / 2$ to $\pi$ the variation range of $L_{t e q}$ is from $L_{t}$ to infinity. That means it is available to adjust $L_{t e q}$ and change the equivalent impedance of the resonant compensation circuit by controlling $\alpha$, thus to adjust $f_{p}$.

The principle of constant frequency control based on dynamic resonance. In order to realize the constant frequency control at the primary side of the system, the inductance phase controlling branch circuit is added to the schematic diagram shown in Fig. 1. The new circuit is shown in Fig. 5 after simplification.

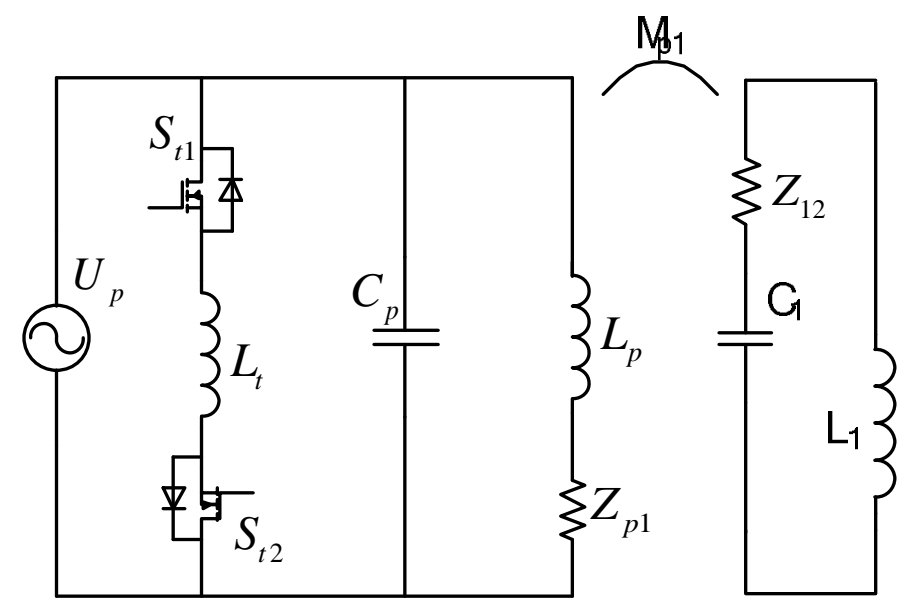

Fig. 5 Equivalent schematic diagram

According to Eq. 1, the following equation can be derived:

$$
Z_{12}=\frac{8 M_{12}^{2} R_{o}}{\pi^{2} M_{2 s}^{2}}
$$

Eq. 8 shows that both $M_{12}$ and $R_{o}$ changes will cause $Z_{12}$ to change. Therefore, the dynamic resonance in the case of the change of $M_{12}$ and $R_{o}$ can be equivalent to the one in the case of the change of $Z_{12}$. Then $Z_{12}$ represents the Varying load of the system shown in Fig. 5.

The admittance of the system shown in Fig. 5 is

$$
G_{P}=\frac{1}{j \omega_{o} L_{t e q}}+j \omega_{o} C_{P}+\frac{1}{j \omega_{o} L_{p}+Z_{p 1}} .
$$

$G_{p 1}$ is the admittance of the primary side resonant network when $L_{t}$ is not considered and can be calculated as

$$
G_{P 1}=j \omega_{o} C_{P}+\frac{1}{j \omega_{o} L_{p}+Z_{p 1}},
$$

where the static compensation capacitor $C_{p}$ is 


$$
C_{p}=\frac{L_{p}}{\omega_{o}^{2} L_{p}^{2}+Z_{p 1}^{2}} .
$$

Since dynamic resonance is based on inductance phase controlling, it is only when the equivalent impedance, after removing $L_{t}$, is capacitive when $Z_{12}$ changes, the phase control inductance has the adjustable range of $f_{p}$. Set $C_{p}$ as

$$
C_{p}=C_{p 1}=\frac{1}{\omega_{o}^{2} L_{p}}=\frac{C_{1} L_{1}}{L_{p}} .
$$

$\operatorname{Im}\left(G_{p 1}\right)=0$ can be obtained when the transmitting coil is in resonance. Substituting $\operatorname{Im}\left(G_{p 1}\right)=0$ to Eq. 12 can give

$$
L_{\text {teq }}=\frac{1}{\omega_{o}^{2} C_{p 1}-\frac{\omega_{o}^{2} L_{p}}{Z_{p 1}^{2}+\omega_{o}^{2} L_{p}^{2}}}=\frac{1}{\omega_{o}^{2} C_{p 1}-\frac{L_{p}}{\frac{\omega_{o}^{2} M_{p 1}^{4}}{Z_{12}^{2}}+L_{p}^{2}}}
$$

According to Eq. 1, the relation curve of $L_{t e q}$ with $Z_{12}$ can be derived as shown in Fig. 6, which shows that the relationship of $L_{t e q}$ and $Z_{12}$ is monotonous. This shows that when the system is in constant frequency control, in the case of $Z_{12}$ changes, $L_{t e q}$ can be adjusted dynamically to $\operatorname{keep} \operatorname{Im}\left(G_{p 1}\right)$ $=0$ valid, thus to keep the inherent resonance frequency of primary side stable.

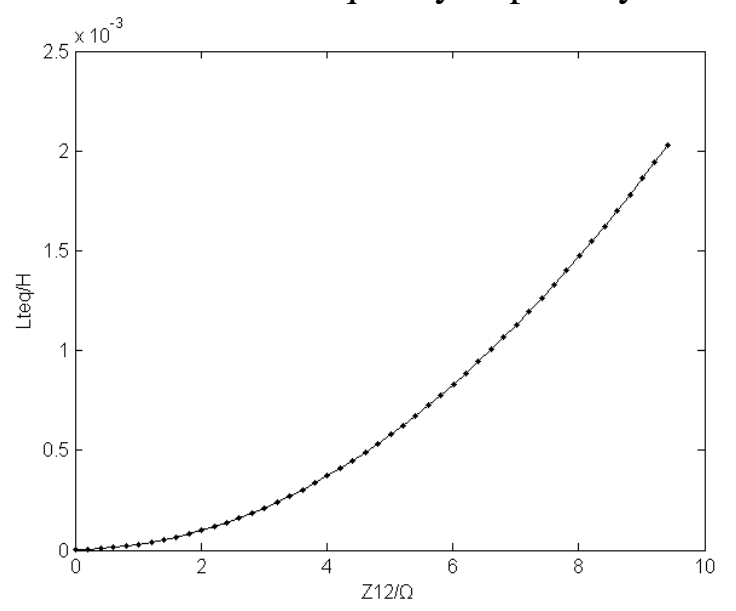

Fig. 6 The relation curve of $L_{t e q}$ with $Z_{12}$

According to Eq. 13, when $Z_{12}$ approaches zero $L_{\text {teq }}$ has a minimum as follows:

$$
L_{\text {teq }(\min )}=\lim _{Z_{12} \rightarrow 0} \frac{1}{\omega_{o}^{2} C_{p 1}-\frac{L_{p}}{\frac{\omega_{o}^{2} M_{p 1}^{4}}{Z_{12}^{2}}+L_{p}^{2}}}=\frac{1}{\omega_{o}^{2} C_{p 1}}=L_{p}
$$

When $Z_{12}$ approaches infinity $L_{t e q}$ has a maximum as follows:

$$
L_{t e q(\max )}=\lim _{Z_{12} \rightarrow \infty} \frac{1}{\omega_{o}^{2} C_{p 1}-\frac{L_{p}}{\frac{\omega_{o}^{2} M_{p 1}^{4}}{Z_{12}^{2}}+L_{p}^{2}}}=\infty
$$

According to Eq. 14 and Eq. 15, when $Z_{12}$ ranges from 0 to infinity, $L_{\text {teq }}$ ranges from $L_{p}$ to infinity. According to the previous conclusion, when $\alpha$ ranges from $\pi / 2$ to $\pi, L_{t e q}$ ranges from $L_{t}$ to infinity. Therefore, to make sure there is always a $\alpha$ to keep $\operatorname{Im}\left(G_{p 1}\right)=0$ valid when $Z_{12}$ changes, the value range of phase control inductance should be: 


$$
L_{t} \leq L_{\text {teq }(\min )}=L_{p}
$$

Constant frequency control strategy based on soft switch technology. The function of the dynamic resonance branch circuit is to make the inherent resonance frequency of primary side consistent with the system operating frequency by adjusting the $L_{\text {teq }}$ to change inherent resonance frequency when they are different. Based on this, this paper proposes a constant frequency control strategy based on soft switch technology. To be more specific, a high frequency inverter controlled by soft switch is adopted at primary side of system to track and sample $f_{p}$, and then to generate corresponding controlling signals to adjust the $L_{\text {teq }}$ to make the inherent resonance frequency of primary side consistent with the system operating frequency by comparing the sample frequency and the rated operating frequency of system. Thus, when $M_{12}$ and $R_{o}$ change, the system can work stably at resonance frequency, and the high frequency inverter of primary side controlled by soft switch is achieved to reduce switch loss at the same time.

According to Eq. 13, the relation curve of $f_{p}$ with $L_{\text {teq }}$ can be derived as shown in Fig. 7

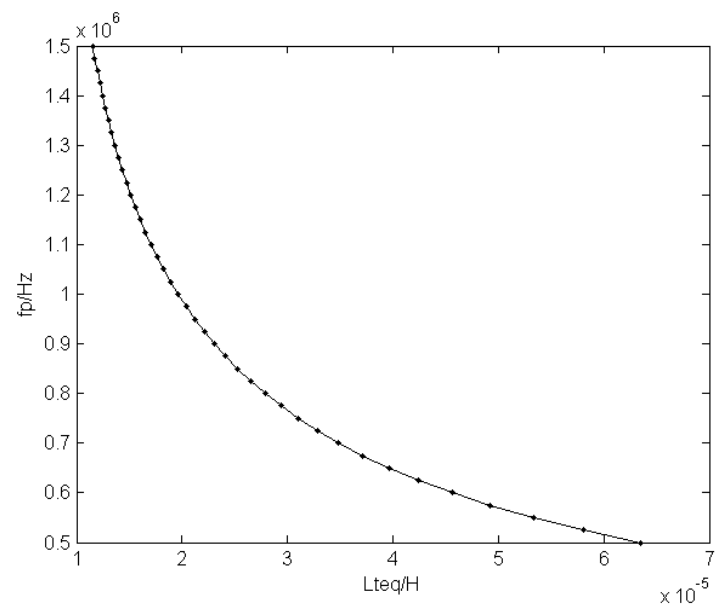

Fig. 7 The relation curve of $f_{p}$ with $L_{t e q}$

According to Eq. 7, Eq. 13 and Fig. 7, the following control logic can be obtained:

(1) When $f=f_{p}>f_{o}$, increase $L_{\text {teq }}$ to increase $\alpha$;

(2) When $f=f_{p}<f_{o}$, decrease $L_{\text {teq }}$ to decrease $\alpha$;

(3) When $f=f_{p}=f_{o}$, just keep $\alpha$ constant.

When the system is under constant frequency control, the waveform of $U_{p}$, drive voltage of the high frequency inverter circuit and drive voltage of the inductance phase controlling branch circuit are shown in Fig. 8. 


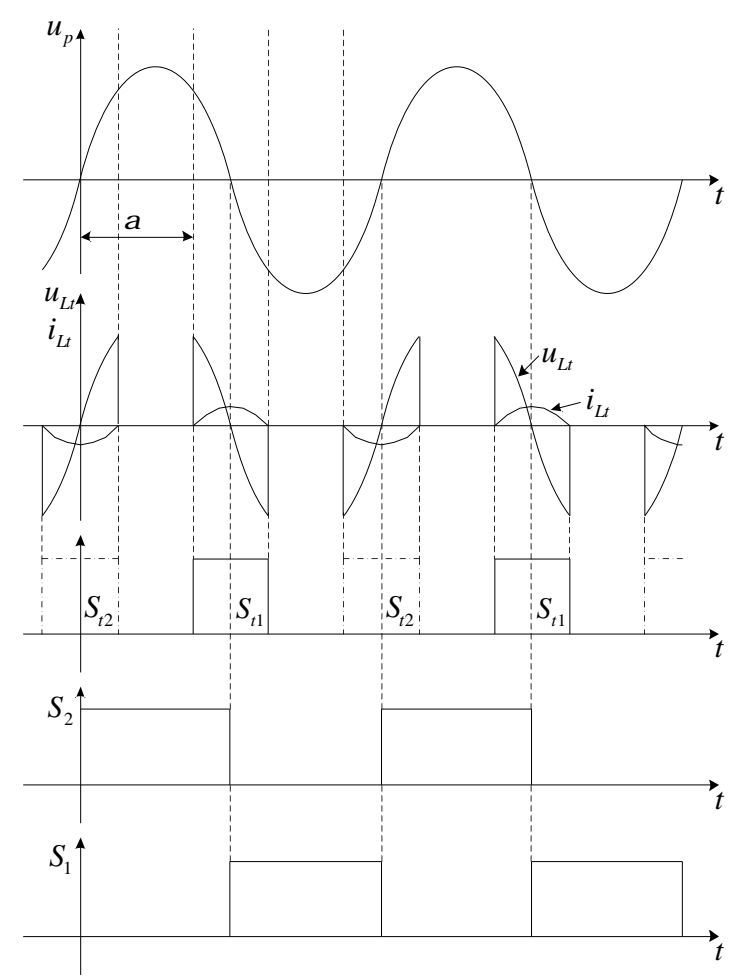

Fig. 8 The waveform of $U_{p}$, drive voltage of the high frequency inverter circuit and drive voltage of the inductance phase controlling branch circuit

Since circuit is not ideal in practice, when switch $S_{t 1}$ or $S_{t 2}$ shut off, the current in the circuit may not reduce to zero, the stray inductance will produce a high reverse voltage the moment the switch shut off, which may lead to damage of the switch. Therefore, it is necessary to extend the turn-on time of switch $S_{t 1}$ and $S_{t 2}$ to make the duty cycle of the switch drive waveform rise to $50 \%$, thus, to make the two drive waveform complementary, so that switch $S_{t 1}$ and $S_{t 2}$ work in zero current switching(ZCS) mode, as shown in Fig. 9.

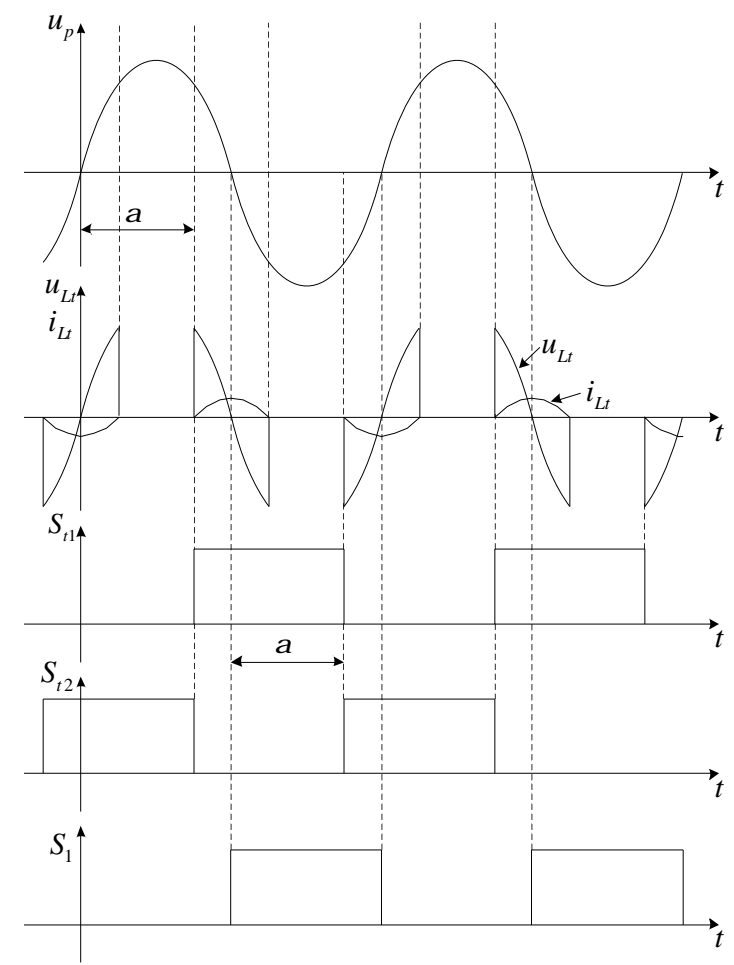

Fig. 9 The waveform of $U_{p}$, drive voltage of the high frequency inverter circuit and drive voltage of the inductance phase controlling branch circuit after improvement 


\section{Simulation Study}

Build the circuit model by using Simulink. Fig. 10 shows the schematic diagram of simulation circuit under constant frequency control, where the high frequency inverter circuit is a push-pull full bridge inverter circuit, $Z_{12}$ represents the varying load when $M_{12}$ and $R_{o}$ change. Table 2 lists the main parameters of the simulation.
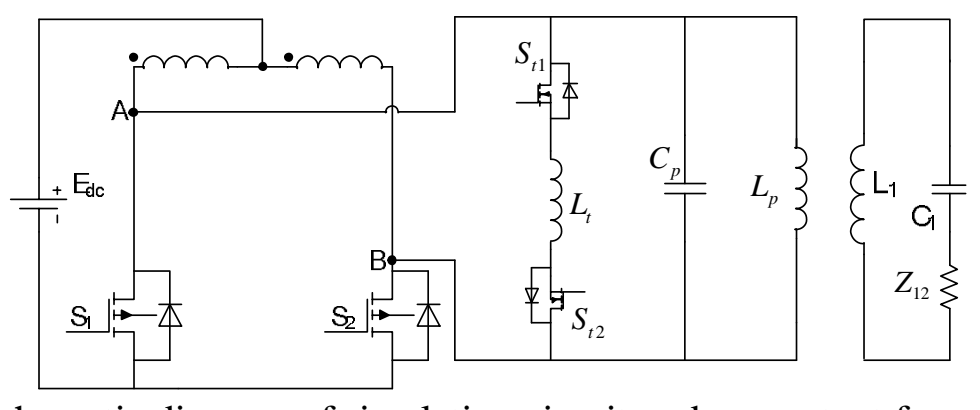

Fig. 10The schematic diagram of simulation circuit under constant frequency control

\begin{tabular}{c|c}
\multicolumn{2}{c}{ Table 2 Simulation Parameters } \\
\hline \hline Parameters & Value \\
\hline$M_{p 1}$ & $0.45[\mu \mathrm{H}]$ \\
\hline$L_{t}$ & $5[\mu \mathrm{H}]$ \\
\hline$L_{p}$ & $5[\mu \mathrm{H}]$ \\
\hline$L_{1}$ & $10[\mu \mathrm{H}]$ \\
\hline$C_{p}$ & $6[\mu \mathrm{F}]$ \\
\hline$C_{1}$ & $5.0661[\mu \mathrm{F}]$ \\
\hline$f$ & $1[\mathrm{MHz}]$ \\
\hline$Z_{12}$ & $10,2[\Omega]$ \\
\hline \hline
\end{tabular}

The simulation time is $0.001 \mathrm{~s}$. In the simulation process, the system firstly works in hard-switching mode with the rated working frequency $f_{o}$; then works in soft-switching mode at $0.0003 \mathrm{~s}$, that is floating frequency control. And the system starts constant frequency control at $0.0004 \mathrm{~s} . Z_{12}$ is adjusted from $10 \Omega$ to $2 \Omega$ at $0.0007 \mathrm{~s}$ and then the simulation is ended in $0.001 \mathrm{~s}$. Fig. 11, Fig. 12 and Fig. 13 show the results of the simulation. Fig. 11 shows the change of system working frequency during the whole simulation process. Fig. 12 and Fig. 13 show respectively the drive voltage waveform of the high frequency inverter circuit, the voltage waveform of the primary resonant network, the voltage and current waveform of phase control inductance and the voltage and current waveform of load when $Z_{12}$ is $10 \Omega$ and $2 \Omega$ after the system starts constant frequency control.

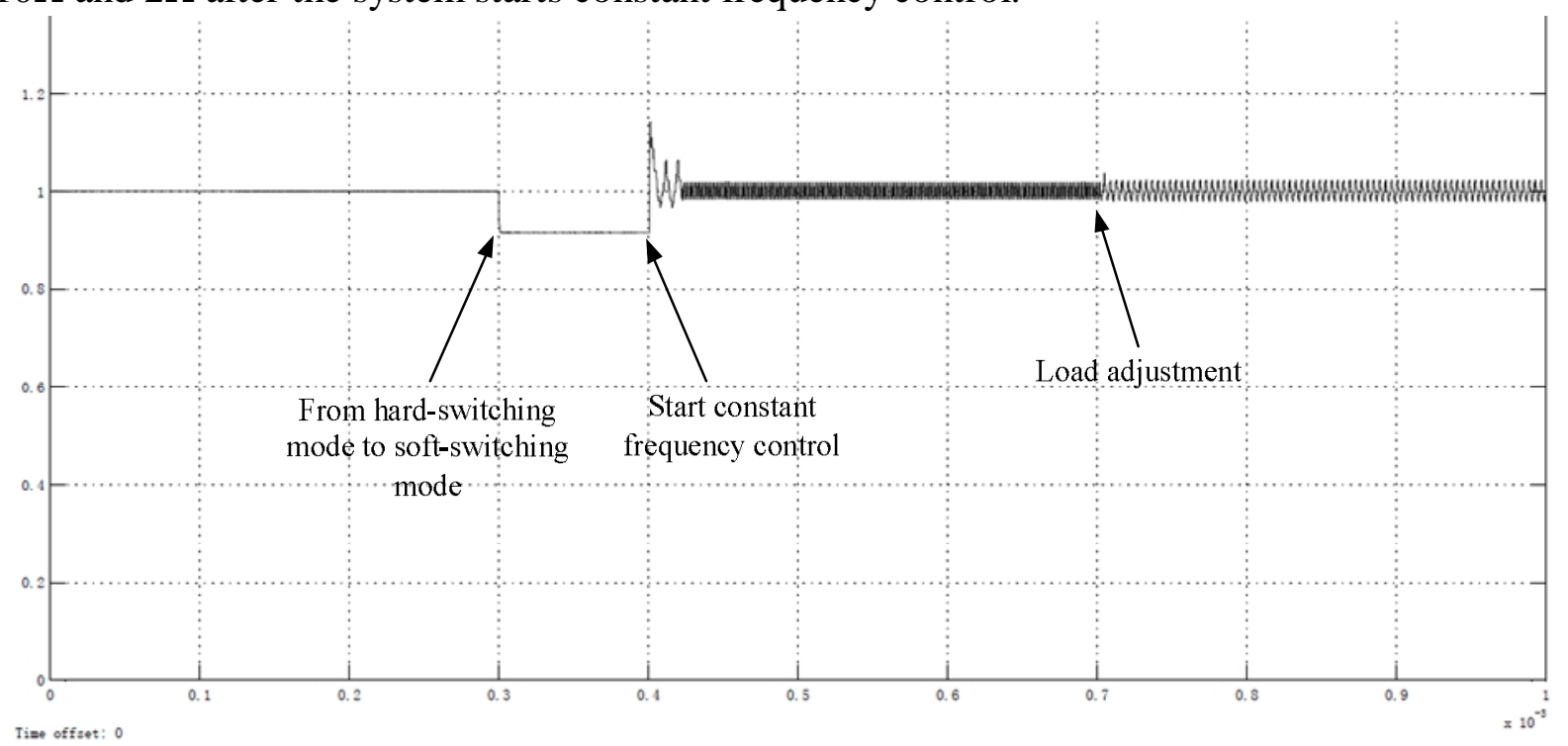

Fig. 11 The change of system working frequency during the whole simulation process 


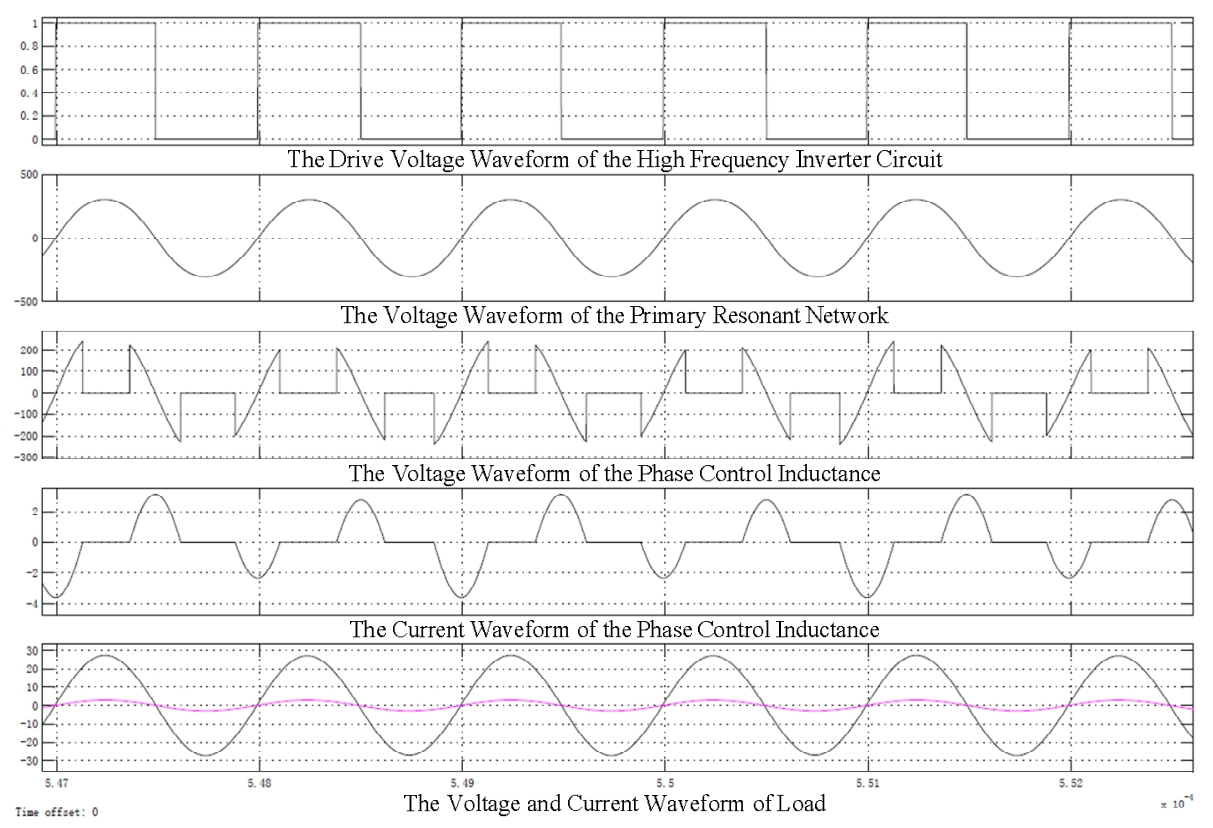

Fig. 12 The drive voltage waveform of the high frequency inverter circuit, the voltage waveform of the primary resonant network, the voltage and current waveform of phase control inductance and the voltage and current waveform of load when $Z_{12}$ is $10 \Omega$
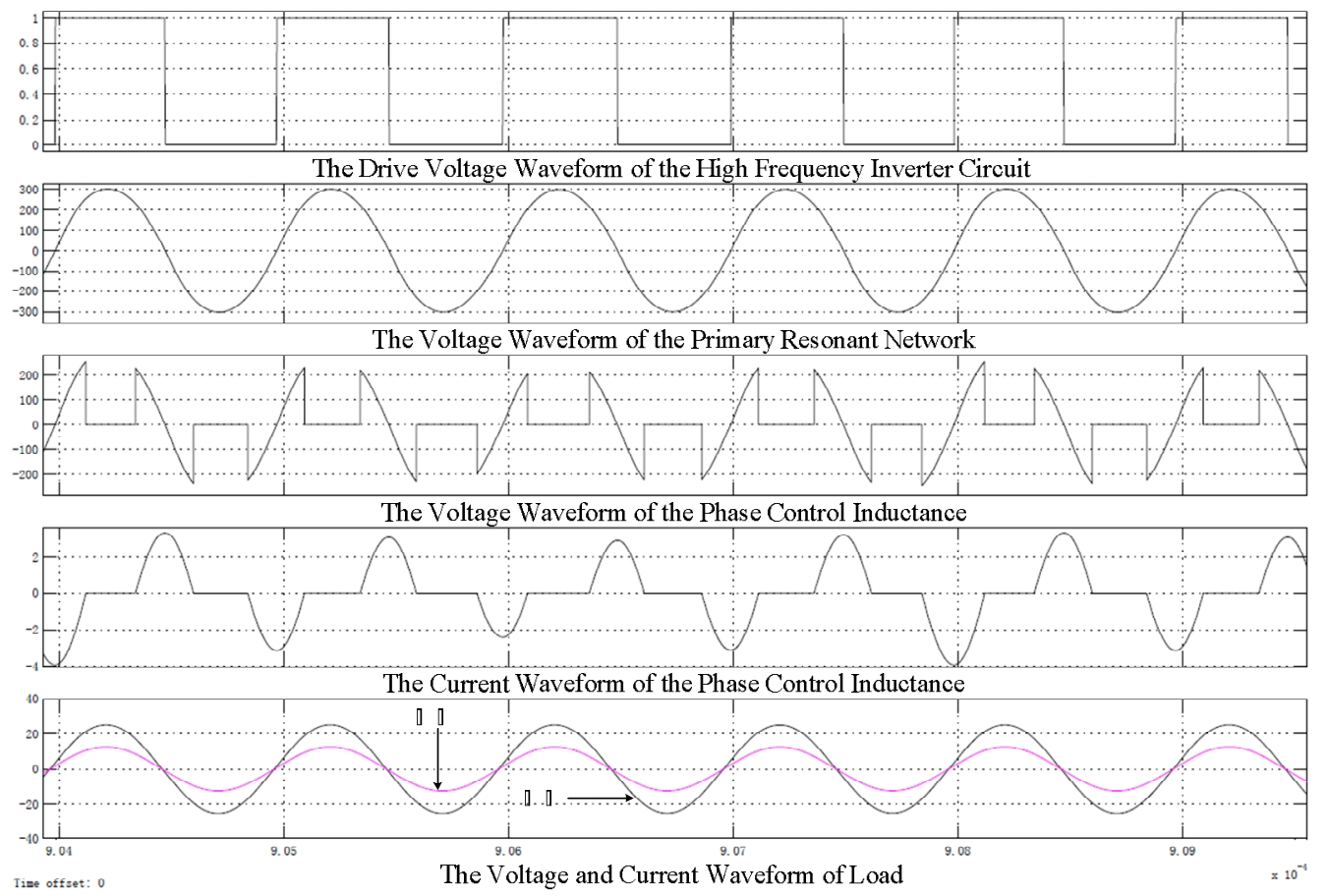

Fig. 13 The drive voltage waveform of the high frequency inverter circuit, the voltage waveform of the primary resonant network, the voltage and current waveform of phase control inductance and the voltage and current waveform of load when $Z_{12}$ is $2 \Omega$

From the simulation results it can be concluded that when $Z_{12}$ changes, the system can work stably at resonance frequency, and the high frequency inverter of primary side works in zero voltage switching (ZVS) mode at the same time.

\section{Conclusion}

This paper, aimed at the magnetic resonance contactless power transmission system based on PSSS topology, analyzes the influence of system load and the coil mutual inductance on the inherent 
resonance frequency of system primary side, proposes the constant frequency control strategy based on dynamic resonance and soft switching technology which achieves the stability of the inherent resonant frequency, and then builds the circuit model by using Simulink and conducts a simulation study. The simulation results show that the constant frequency control strategy is feasible.

\section{References}

[1] Boys J T, Hu A P, Covic G A. Critical Q Analysis of a Current-fed Resonant Converter for ICPT Applications[J]. Electronics Letters, 2000, 36(17): 1440-1442.

[2] André Kurs, Aristeidis Karalis, Robert Moffatt, et al. Wireless Power Transfer via Strongly Coupled Magnetic Resonances[J]. Science, 2007, 317(5834): 83 - 86.

[3] Yuan Zhai. Study on Optimization and Control of Magnetic Resonance Mode Wireless Power Supply System[D]. Chongqing University. 2013.

[4] Yu Gang Su, Chun Sen Tang, Yue Sun, Zhi Hui Wang. Load Adaptive Technology of Contactless Power Transfer System[J]. Transaction of China Electrotechnical Society, 2009, 24(1): 153-157.

[5] Chen Yang Xia. Research on Analysis and Optimization for Transmission Power and Efficiency of Inductively Coupled Power Transfer System[D]. Chongqing University. 2010.

[6] Chun Sen Tang, Yue Sun, Yu Gang Su, et al. Determining multiple steady-state ZCS operating points of a switch-mode contactless power transfer system [J]. IEEE Transactions On Power Electronics, 2009, 24(2): 416-425.

[7] Chwei-Sen Wang, Grant A. Covic, Oskar H. Stielau. Power Transfer Capability and Bifurcation Phenomena of Loosely Coupled Inductive Power Transfer Systems [J]. IEEE Transactions On Power Electronics, 2004, 51(1): 148-157. 